\title{
A New Scheme for the Design of Hilbert Transform Pairs of Biorthogonal Wavelet Bases
}

\author{
Hongli Shi and Shuqian Luo \\ College of Biomedical Engineering, Capital Medical University, Beijing 100069, China \\ Correspondence should be addressed to Hongli Shi, shl@ccmu.edu.cn
}

Received 29 August 2010; Accepted 10 November 2010

Academic Editor: Ulrich Heute

Copyright () 2010 H. Shi and S. Luo. This is an open access article distributed under the Creative Commons Attribution License, which permits unrestricted use, distribution, and reproduction in any medium, provided the original work is properly cited.

In designing the Hilbert transform pairs of biorthogonal wavelet bases, it has been shown that the requirements of the equalmagnitude responses and the half-sample phase offset on the lowpass filters are the necessary and sufficient condition. In this paper, the relationship between the phase offset and the vanishing moment difference of biorthogonal scaling filters is derived, which implies a simple way to choose the vanishing moments so that the phase response requirement can be satisfied structurally. The magnitude response requirement is approximately achieved by a constrained optimization procedure, where the objective function and constraints are all expressed in terms of the auxiliary filters of scaling filters rather than the scaling filters directly. Generally, the calculation burden in the design implementation will be less than that of the current schemes. The integral of magnitude response difference between the primal and dual scaling filters has been chosen as the objective function, which expresses the magnitude response requirements in the whole frequency range. Two design examples illustrate that the biorthogonal wavelet bases designed by the proposed scheme are very close to Hilbert transform pairs.

\section{Introduction}

The dual-tree complex wavelet transform (DTCWT) had become an attractive signal processing tool since it was proposed by Kingsbury [1, 2]. It overcomes the main drawbacks of real discrete wavelet transform (DWT), such as shift sensitivity and poor directional selectivity (in the case of multidimensional DWT). A pair of filter banks is used in DTCWT, with which the wavelet bases associated form (approximate) Hilbert transform pairs. This property is critical since it is vital to reduce shift sensitivity and improve directionality [3].

Selesnick [4], and Ozkaramanli and Yu [5, 6] had studied the conditions under which the corresponding orthogonal wavelet bases form Hilbert transform pairs. It shows that if and only if one orthogonal low-pass filter is half-sample delayed from the other, the corresponding wavelets form a Hilbert transform pair. The compactly supported real orthogonal wavelets (except Haar wavelet) cannot be of linear phase (symmetry), however, linear phase is essential in many signal processing applications. As a result, the biorthogonal wavelets are usually suggested to be used, for example, JPEG2000 standard. Yu and Ozkaramanli [7] had further proven that the requirements of half-sample offset are also the necessary and sufficient condition for the design of Hilbert transform pairs of biorthogonal wavelet bases.

In [8], Selesnick proposed a new design scheme and designed two filter banks that the corresponding wavelets forming approximate Hilbert transform pairs. In [9], the design is formulated as a sampled-data $H^{\infty}$ optimization problem (suppose the primal filters are known) and then solved by standard approximation theory. The design scheme usually gives infinite impulse response (IIR) filters, which have to be truncated to form FIR filters. As a result, the dual filter bank is not symmetric and of perfect reconstruction (PR). In [7], the design is achieved by a constrained optimization procedure, which can ensure the filter bank to be symmetrical and PR. In the design, the objective function and constraints are all expressed in terms of the lowpass filters, that is, $h_{0}, \tilde{h}_{0}, g_{0}$, and $\tilde{g}_{0}$ (see Section 2 for the notations). The objective function in [7] is equivalent to a $L^{\infty}$ 


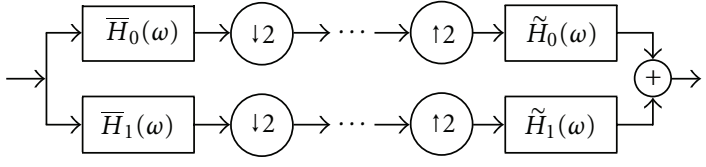

FIGURE 1: Filter bank structure of primal biorthogonal wavelet.

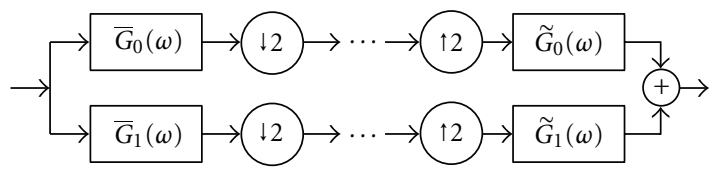

FIGURE 2: Filter bank structure of dual biorthogonal wavelet.

measure, which involves a constrained minimax procedure. A series of objective functions are formed by selecting certain frequencies in $[-\pi, \pi]$, which express the magnitude response requirements at these discrete frequencies.

In this paper, a new design scheme is presented and the design implementation is described in detail. We have derived a relationship between the phase offset and the vanishing moment difference of two biorthogonal low-pass filters, which can be used to choose the vanishing moments so that the phase requirement is satisfied structurally. The design is also achieved by a constrained optimization procedure, in which the objective function and constraints are all expressed in terms of the auxiliary filters $\left(p_{h}, p_{\tilde{h}}\right.$, $p_{g}$, and $\left.p_{\tilde{g}}\right)$ rather than the low-pass filters directly. When the low-pass filters are of high vanishing moments in total, the variable number can be reduced. The objective function chosen is equivalent to a $L^{2}$ measure, which expresses the magnitude response requirements in the whole frequency range. Therefore, a better approximation quality to Hilbert transform pair is possible.

\section{Preliminaries}

2.1. Biorthogonal Wavelet. Suppose that $H_{0}(z)=$ $\sum_{n} h_{0}(n) z^{-n}$ and $\tilde{H}_{0}(z)=\sum_{n} \widetilde{h}_{0}(n) z^{-n}$ represent the biorthogonal low-pass filters in the decomposition and reconstruction sides in Figure 1, respectively. $H_{1}(z)$ and $\tilde{H}_{1}(z)$ represent the associated highpass filters. $\psi_{h}(t)$ and $\widetilde{\psi}_{h}(t)$ denote the corresponding wavelet bases. By choosing $H_{1}(z)=z \overline{\widetilde{H}}_{0}(-z)$ and $\widetilde{H}_{1}(z)=z \bar{H}_{0}(-z)$, the no-distortion and no-aliasing conditions require that $[10]$

$$
H_{0}(z) \overline{\tilde{H}}_{0}(z)+H_{0}(-z) \overline{\widetilde{H}}_{0}(-z)=2 .
$$

Equation (1) expresses the PR requirement of biorthogonal filter bank. In terms of the filter coefficients, it becomes

$$
\sum_{n} h_{0}(n) \tilde{h}_{0}(n+2 k)=2 \delta_{k, 0}
$$

In order to obtain smooth biorthogonal wavelet bases, certain numbers of zeros at $z=-1$ for $H_{0}(z)$ and $\tilde{H}_{0}(z)$, are always imposed; that is, the filters have certain numbers of vanishing moments [10]. Suppose that $H_{0}(z)=(1 / \sqrt{2})(1+$ $\left.z^{-1}\right)^{k_{h}} p_{h}(z), \widetilde{H}_{0}(z)=(1 / \sqrt{2})\left(1+z^{-1}\right)^{\widetilde{k}_{h}} \widetilde{p}_{h}(z)$, where $p_{h}(z)$ and $\tilde{p}_{h}(z)$ each denote real coefficient symmetric filters, $p_{h}(-1) \neq 0, \tilde{p}_{h}(-1) \neq 0, k_{h} \in \mathbb{Z}^{+}$, and $\tilde{k}_{h} \in \mathbb{Z}^{+} . p_{h}(z)$ and $\tilde{p}_{h}(z)$ are known as the auxiliary filters of $H_{0}(z)$ and $\tilde{H}_{0}(z)$ [11], respectively. $h_{0}$ and $\widetilde{h}_{0}$ are either both of odd length or both even length, (1) can be written as [12]

$$
\begin{aligned}
& \left(\cos \frac{\omega}{2}\right)^{2 K_{h}} P_{h}(\cos \omega) \overline{\widetilde{P}_{h}(\cos \omega)} \\
& \quad+\left(\sin \frac{\omega}{2}\right)^{2 K_{h}} P_{h}(-\cos \omega) \overline{\widetilde{P}_{h}(-\cos \omega)}=1,
\end{aligned}
$$

where $K_{h}=\left(k_{h}+\tilde{k}_{h}\right) / 2$ (it means the vanishing moments of $h_{0}$ and $\tilde{h}_{0}$ must be either both odd or both even) and $P_{h}(\cos \omega)$ and $\widetilde{P}_{h}(\cos \omega)$ represent the discrete time Fourier transform (DTFT) of $p_{h}$ and $\tilde{p}_{h}$, respectively. $P_{h}(\cos \omega)$ and $\widetilde{P}_{h}(\cos \omega)$ all can be expressed as real polynomials in terms of $\cos \omega$ by introducing suitable integer translations. Cohen et al. [12] had given the solution of (3), that is,

$$
\begin{aligned}
& P_{h}(\cos \omega) \overline{\widetilde{P}_{h}(\cos \omega)} \\
& \quad=P_{K_{h}}\left(\sin ^{2} \frac{\omega}{2}\right)+\left(\sin ^{2} \frac{\omega}{2}\right)^{K_{h}} R_{h}\left(1-2 \sin ^{2} \frac{\omega}{2}\right),
\end{aligned}
$$

where $R_{h}(\cdot)$ is an odd polynomial, and

$$
P_{K_{h}}\left(\sin ^{2} \frac{\omega}{2}\right)=\sum_{n=0}^{K_{h}-1}\left(\begin{array}{c}
K_{h}-1+n \\
n
\end{array}\right) \sin ^{2 n} \frac{\omega}{2} .
$$

Equation (4) is equivalent to (1) or (2), which is the foundation in designing biorthogonal wavelet.

2.2. Hilbert Transform Pairs of Biorthogonal Wavelets. Suppose the corresponding decomposition wavelet $\psi_{g}(t)$ in Figure 2 to be the Hilbert transform of $\psi_{h}(t)$ in Figure 1, and the reconstruction wavelet $\widetilde{\psi}_{h}(t)$ to be the Hilbert transform of $\widetilde{\psi}_{g}(t)$. That is

$$
\psi_{g}(t)=\mathscr{H}\left\{\psi_{h}(t)\right\}, \quad \tilde{\psi}_{h}(t)=\mathscr{H}\left\{\tilde{\psi}_{g}(t)\right\},
$$

that is,

$$
\begin{aligned}
& \Psi_{g}(\omega)= \begin{cases}-i \Psi_{h}(\omega), & \omega>0, \\
i \Psi_{h}(\omega), & \omega<0,\end{cases} \\
& \widetilde{\Psi}_{h}(\omega)= \begin{cases}-i \widetilde{\Psi}_{g}(\omega), & \omega>0, \\
i \widetilde{\Psi}_{g}(\omega), & \omega<0,\end{cases}
\end{aligned}
$$

where $\Psi_{h}(\omega), \Psi_{g}(\omega), \widetilde{\Psi}_{h}(\omega)$, and $\widetilde{\Psi}_{g}(\omega)$ denote the Fourier transforms of $\psi_{h}(t), \psi_{g}(t), \widetilde{\psi}_{h}(t)$, and $\widetilde{\psi}_{g}(t)$, respectively. The filter bank in Figure 2 is named as the dual filter bank of that in Figure 1 [7]. $h_{0}$ and $\tilde{h}_{0}$ are known as the primal low-pass filters, and $g_{0}$ and $\tilde{g}_{0}$ are known as the dual low-pass filters. 


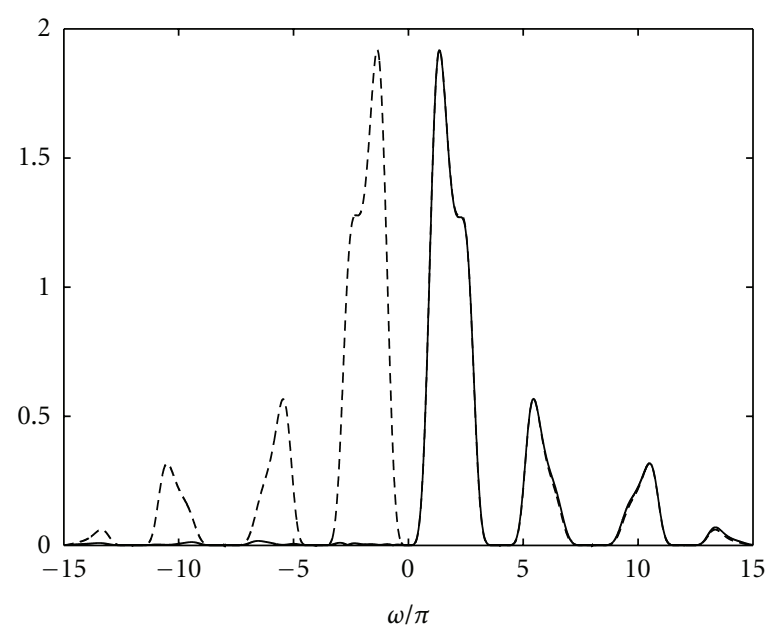

(a)

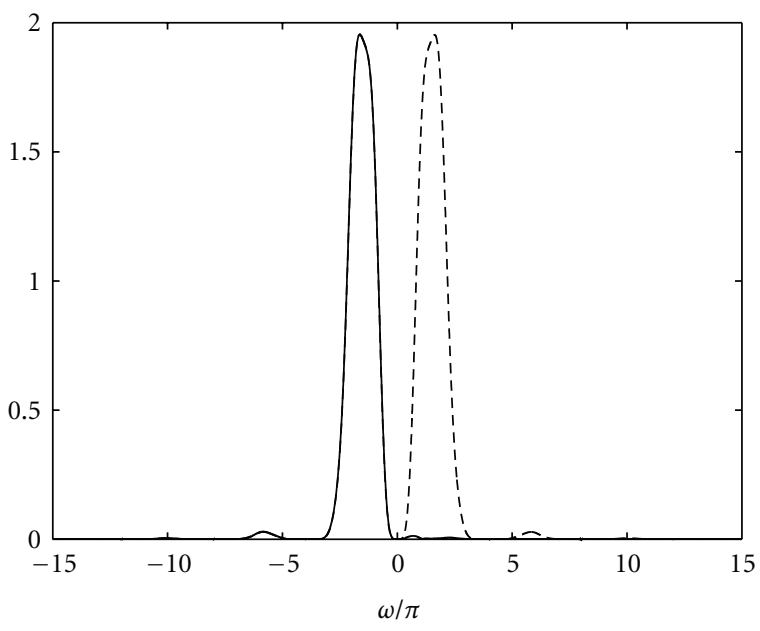

(b)

FIGURE 3: Magnitude responses of wavelet bases in Example 1. (a) Magnitude responses of decomposition wavelets: $\left|\Psi_{h}(\omega)+i \Psi_{g}(\omega)\right|($ the solid line) and $2\left|\Psi_{h}(\omega)\right|$ (the dashed line). (b) Magnitude responses of reconstruction wavelets: $\left|\widetilde{\Psi}_{h}(\omega)+i \widetilde{\Psi}_{g}(\omega)\right|\left(\right.$ the solid line) and $2\left|\widetilde{\Psi}_{h}(\omega)\right|$ (the dashed line).

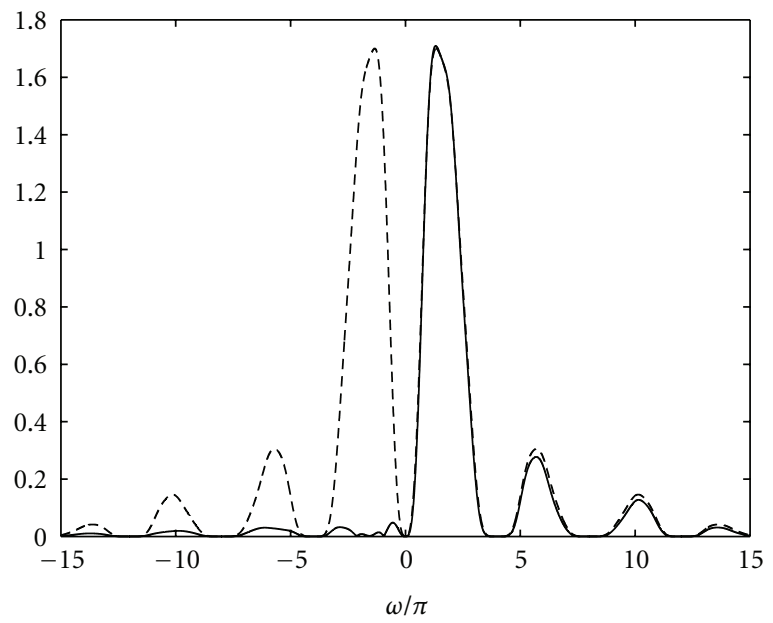

(a)

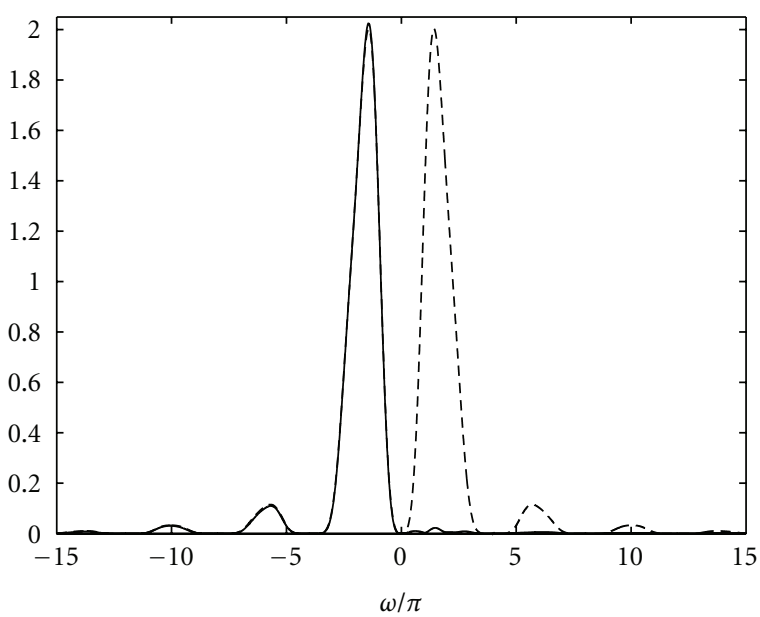

(b)

Figure 4: Magnitude responses of wavelet bases in Example 2. (a) Magnitude responses of decomposition wavelets: $\left|\Psi_{h}(\omega)+i \Psi_{g}(\omega)\right|($ the solid line) and $2\left|\Psi_{h}(\omega)\right|$ (the dashed line). (b) Magnitude responses of reconstruction wavelets: $\left|\widetilde{\Psi}_{h}(\omega)+i \widetilde{\Psi}_{g}(\omega)\right|($ the solid line $)$ and $2\left|\widetilde{\Psi}_{h}(\omega)\right|$ (the dashed line).

Table 1: The filter coefficients in Example 1.

\begin{tabular}{lccccccc}
\hline$n$ & $h_{0}$ & $n$ & $\tilde{h}_{0}$ & $n$ & $g_{0}$ & $n$ & $\tilde{g}_{0}$ \\
\hline$-5,6$ & -0.003744218100161 & $-5,6$ & 0.001973944277540 & $-6,6$ & -0.002679477007908 & $-5,5$ & 0.006291060002458 \\
$-4,5$ & 0.027155595407045 & $-4,5$ & 0.014316375468255 & $-5,5$ & 0.007973992387869 & $-4,4$ & 0.018721886555915 \\
$-3,4$ & 0.001335375233427 & $-3,4$ & 0.015452878287554 & $-4,4$ & 0.036852253064238 & $-3,3$ & 0.006861425697625 \\
$-2,3$ & -0.207583964407648 & $-2,3$ & 0.007742782192903 & $-3,3$ & -0.097728862384778 & $-2,2$ & 0.048456665186341 \\
$-1,2$ & -0.036015217586016 & $-1,2$ & 0.143297943721683 & $-2,2$ & -0.216559663541172 & $-1,1$ & 0.274347514299916 \\
0,1 & 0.653635038149005 & 0,1 & 0.392216076052064 & $-1,1$ & 0.307146174344736 & 0 & 0.440642896515488 \\
& & & & 0 & 0.799556383665335 & & \\
\hline
\end{tabular}




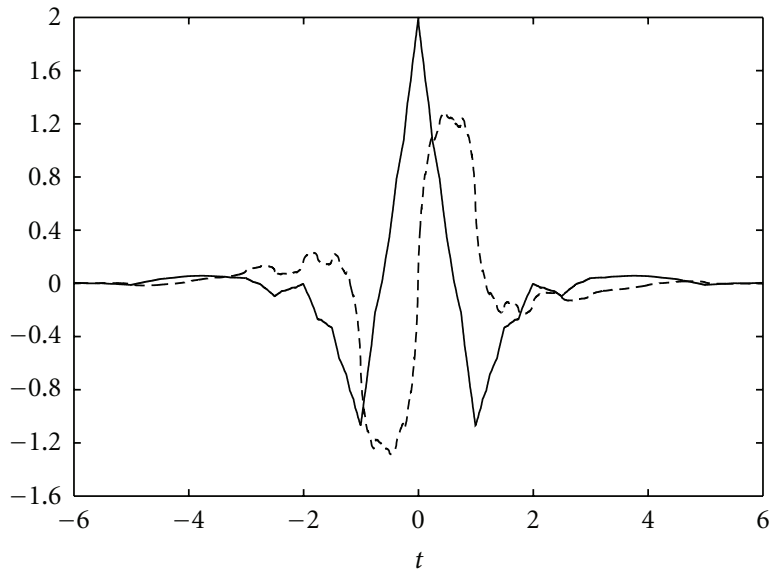

(a)

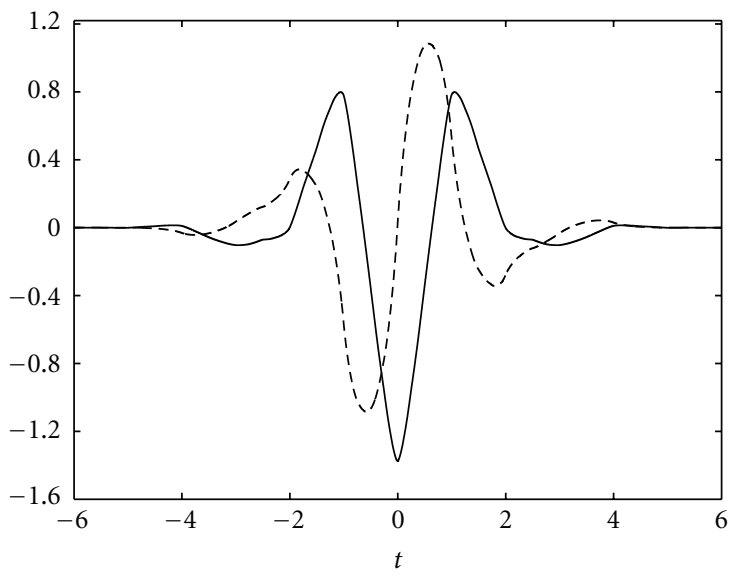

(b)

Figure 5: Wavelet bases in Example 2. (a) Decomposition wavelets: $\psi_{h}(t)$ of Antonini 9/7 wavelets (the solid line) and $\psi_{g}(t)$ of dual wavelets (the dashed line). (b) Reconstruction wavelets: $\widetilde{\psi}_{h}(t)$ of Antonini 9/7 wavelets (the solid line) and $\widetilde{\psi}_{g}(t)$ of dual wavelets (the dashed line).

Yu and Ozkaramanli had proven that if and only if the primal and dual low-pass filters satisfy

$$
G_{0}(\omega)=e^{-j \omega / 2} H_{0}(\omega), \quad \widetilde{G}_{0}(\omega)=e^{j \omega / 2} \tilde{H}_{0}(\omega), \quad|\omega|<\pi,
$$

the corresponding biorthogonal wavelets satisfy (6), where $H_{0}(\omega), G_{0}(\omega), \tilde{H}_{0}(\omega)$, and $\widetilde{G}_{0}(\omega)$ denote DTFTs of $h_{0}, g_{0}$, $\tilde{h}_{0}$, and $\tilde{g}_{0}$, respectively. The phase requirement in (8) can be satisfied by choosing the vanishing moments of the filters in a simple way, which will be described in the next section. However, the requirement of magnitude responses has to be approximately achieved by an optimization procedure. The optimization routine in [7] is simplified as follows:

$$
\begin{array}{ll}
\max _{|\omega|<\pi} & \left\{\left\|G_{0}(\omega)-e^{-j w / 2} H_{0}(\omega)\right\|^{2} \Longrightarrow \min \right. \\
& \left.+\left\|\widetilde{G}_{0}(\omega)=\widetilde{H}_{0}(\omega) e^{j \omega / 2}\right\|^{2}\right\} \\
& \sum_{n} h_{0}(n) \widetilde{h}_{0}(n+2 k)=2 \delta_{k, 0} . \\
\text { st. } \quad & \sum_{n} 9_{0}(n) \tilde{g}_{0}(n+2 k)=2 \delta_{k, 0} .
\end{array}
$$

(In the design example, Antonini 9/7 filters are chosen as $h_{0}$ and $\widetilde{h}_{0}$, and the constraintis $\sum_{n} h_{0}(n) \tilde{h}_{0}(n+2 k)=2 \delta_{k, 0}$ are no longer necessary).

The optimization routine (9) involves $\left\lfloor\left(L_{h}+1\right) / 2\right\rfloor+\left\lfloor\left(\widetilde{L}_{h}+\right.\right.$ $1) / 2\rfloor+\left\lfloor\left(L_{g}+1\right) / 2\right\rfloor+\left\lfloor\left(\widetilde{L}_{g}+1\right) / 2\right\rfloor$ variables, where $\lfloor x\rfloor$ denotes the greatest integer not exceeding $x$ and $L_{h}, \tilde{L}_{h}, L_{g}$, and $\tilde{L}_{g}$ denote the lengths of $h_{0}, \widetilde{h}_{0}, g_{0}$, and $\widetilde{h}_{0}$, respectively. The design scheme (9) involves a constrained minimax routine. In the design, a series of objective functions are formed by choosing certain frequencies, which express the magnitude response requirements at discrete frequencies. Since the low-pass filters can be represented by their auxiliary filters uniquely, the optimization routine can be expressed by the auxiliary filters uniquely. If the filters have high vanishing moments in total, the variable number of objective function and constraints can be reduced. As a result, the calculation burden in optimization routine can be reduced. Furthermore, the objective function can be chosen to express the magnitude response requirement in whole frequency range.

\section{A New Design Scheme}

The new design scheme is also based on the sufficient and necessary conditions that give Hilbert transform pairs of biorthogonal wavelet base, that is, (8). First of all, the biorthogonal filter banks should be designed to satisfy PR requirement.

A biorthogonal low-pass filter, $H(z)$, is either of odd length or even length, and it can be always expressed as $H(z)=(1 / \sqrt{2})\left(1+z^{-1}\right)^{k} p(z)$ with $p(z)(2 l+1)$-length symmetric filter, $l, k \in \mathbb{Z}^{+}, p(-1) \neq 0$ (if $p(z)$ is a symmetric filter of $(2 l+2)$-tap, it can be expressed as $p(z)=(1+$ $\left.z^{-1}\right) p^{\prime}(z)$ with $p^{\prime}(z)$ being a symmetric filter of $(2 l+1)$ tap). Let $H_{0}(z)=(1 / \sqrt{2})\left(1+z^{-1}\right)^{k_{h}} p_{h}(z)$ denote the primal low-pass filter with $p_{h}(z)$ a $\left(2 l_{h}+1\right)$-tap symmetric filter. By introducing a suitable integer translation, $p_{h}(z)$ is expressed as $p_{h}(z)=p_{h, 0}+\sum_{k=1}^{l_{h}} p_{h, k}\left(z^{k}+z^{-k}\right)=P_{h}(\cos \omega)$. Similarly, $\tilde{H}_{0}(z)=(1 / \sqrt{2})\left(1+z^{-1}\right)^{\tilde{k}_{h}} \widetilde{p}_{h}(z)$ with $\widetilde{p}_{h}(z)$ a $\left(2 \tilde{l}_{h}+1\right)$-tap being symmetric filter, $\widetilde{p}_{h}(z)=\widetilde{p}_{h, 0}+\sum_{k=1}^{\widetilde{l}_{h}} \widetilde{p}_{h, k}\left(z^{k}+z^{-k}\right)=$ $\widetilde{P}_{h}(\cos \omega)$. Since $z=e^{-j \omega}, \sin ^{2}(\omega / 2)=1 / 2-\left(z+z^{-1}\right) / 4$, according to (4), we have

$$
\begin{aligned}
p_{h}(z) \overline{\tilde{p}_{h}(z)}= & P_{h}(\cos \omega) \overline{\widetilde{P}_{h}(\cos \omega)} \\
= & \sum_{n=0}^{K_{h}-1}\left(\begin{array}{c}
K_{h}-1+n \\
n
\end{array}\right)\left(\frac{1}{2}-\frac{z+z^{-1}}{4}\right)^{n} \\
& +\left(\frac{1}{2}-\frac{z+z^{-1}}{4}\right)^{K_{g}} R_{h}\left(\frac{z+z^{-1}}{2}\right),
\end{aligned}
$$


TABLE 2: $E_{1}$ and $E_{2}$ measures in literature [7], Example 1 and Example 2.

\begin{tabular}{|c|c|c|c|c|c|c|}
\hline \multirow{2}{*}{ Filters } & \multicolumn{3}{|c|}{$E_{1}$} & \multicolumn{3}{|c|}{$E_{2}$} \\
\hline & De. & Re. & Av. & De. & Re. & Av. \\
\hline Literature [7] & 0.0229 & 0.0213 & 0.0221 & $4.855 E-4$ & $3.715 E-4$ & $4.285 E-4$ \\
\hline Example 1 & 0.0074 & 0.0088 & 0.0081 & $5.195 E-5$ & $3.944 E-5$ & $4.5693 E-5$ \\
\hline Example 2 & 0.0109 & 0.0237 & 0.0173 & $2.2563 E-4$ & $5.9078 E-4$ & $4.0821 E-4$ \\
\hline
\end{tabular}

*: "De." means "Decomposition wavelet"; "Re." means "Reconstruction wavelet"; "Av." means "Average value".

TABLE 3: The coefficients of low-pass filters in Example 2.

\begin{tabular}{lccc}
\hline \multicolumn{4}{c}{ The filter lengths are of $10 / 10$ with $k_{g}=\widetilde{k}_{g}=3$} \\
$n$ & $g_{0}$ & $n$ & $\widetilde{g}_{0}$ \\
\hline$-4,5$ & 0.005177090817695 & $-5,4$ & 0.003154113122922 \\
$-3,4$ & 0.021637688714158 & $-4,3$ & -0.013182638730184 \\
$-2,3$ & -0.062344308626311 & $-3,2$ & -0.064516826255491 \\
$-1,2$ & 0.030709855881950 & $-2,1$ & 0.092188868846268 \\
0,1 & 0.508721114417255 & $-1,0$ & 0.478485248598678 \\
\hline
\end{tabular}

where $K_{h}=\left(k_{h}+\tilde{k}_{h}\right) / 2 ; R_{h}(\cdot)$ is an odd polynomial. Similarly,

$$
\begin{aligned}
p_{g}(z) \overline{\tilde{p}_{g}(z)}= & \sum_{n=0}^{K_{g}-1}\left(\begin{array}{c}
K_{g}-1+n \\
n
\end{array}\right)\left(\frac{1}{2}-\frac{z+z^{-1}}{4}\right)^{n} \\
& +\left(\frac{1}{2}-\frac{z+z^{-1}}{4}\right)^{K_{g}} R_{g}\left(\frac{z+z^{-1}}{2}\right),
\end{aligned}
$$

where $K_{g}=\left(k_{g}+\tilde{k}_{g}\right) / 2 ; R_{g}(\cdot)$ is an odd polynomial. Since $p_{h}(z) \overline{\tilde{p}_{h}(z)}$ is symmetric and has $\left(2 l_{h}+2 \tilde{l}_{h}+1\right)$ taps, we can derive $\left(l_{h}+\tilde{l}_{h}+1\right)$ constraints on the filter $p_{h}$ and $\tilde{p}_{h}$ and the polynomial coefficients of $R_{h}(\cdot)$ according to (10). Similarly, we can derive $\left(l_{g}+\tilde{l}_{g}+1\right)$ constraints on $p_{g}$ and $\tilde{p}_{g}$ and $R_{g}(\cdot)$ from (11).

Equations (10) and (11) ensure that the filters $H_{0}(z)$ and $\tilde{H}_{0}(z), G_{0}(z)$, and $\widetilde{G}_{0}(z)$ satisfy $\mathrm{PR}$ requirement, respectively. If $\left(2 l_{h}+2 \tilde{l}_{h}+2\right)>\left(k_{h}+\tilde{k}_{h}\right)$, that is, the polynomial $R_{h}(\cdot) \neq 0, p_{h}(z) \overline{p_{\bar{h}}(z)}$ is not unique, and so are $h_{0}(z)$ and $\tilde{h}_{0}(z)$. Similarly, if $R_{g}(\cdot) \neq 0, g_{0}(z)$ and $\tilde{g}_{0}(z)$ are not unique. Therefore, it is possible to construct biorthogonal filter banks that satisfy the PR requirement in addition to approximately satisfying $(8)$ when $R_{h}(\cdot) \neq 0$ and/or $R_{g}(\cdot) \neq 0$.

For the requirement of half-sample offset, first consider the requirement of phase group delay/advance. The relationship between the phase offset and the vanishing moment difference of two biorthogonal low-pass filters can be characterized by the following proposition.

Proposition 1. Supposing two biorthogonal low-pass filters, $H(z)=(1 / \sqrt{2})\left(1+z^{-1}\right)^{k_{1}} p_{1}(z)$ and $G(z)=(1 / \sqrt{2})(1+$ $\left.z^{-1}\right)^{k_{2}} p_{2}(z)$, where $p_{1}(z)$ and $p_{2}(z)$ each indicate symmetric FIR filters, $p_{1}(-1) \neq 0, p_{2}(-1) \neq 0$, then

$$
\angle H(\omega)-\angle G(\omega)=k \omega+\frac{\omega}{2}\left(k_{2}-k_{1}\right),
$$

where $\angle$ denotes the phase angle; $l \in \mathbb{Z}$.
Proof. Since $p_{1}(z)$ and $p_{2}(z)$ each are symmetric and of odd length, they can be expressed as

$$
\begin{aligned}
& P_{1}(\cos \omega)=e^{-j l_{1} \omega} p_{1}^{\prime}(\cos \omega), \\
& P_{2}(\cos \omega)=e^{-j l_{2} \omega} p_{2}^{\prime}(\cos \omega),
\end{aligned}
$$

where $p_{1}^{\prime}(\cos \omega)$ and $p_{2}^{\prime}(\cos \omega)$ each are real polynomials in terms of $\cos \omega ; l_{1} \in \mathbb{Z}, l_{2} \in \mathbb{Z}$. Since $\left(1+e^{-j \omega}\right)=$ $2 \cos (\omega / 2) e^{-j \omega / 2}$, it results in

$$
\angle H(\omega)=-\left(l_{1} \omega+\frac{\omega}{2} k_{1}\right), \quad \angle G(\omega)=-\left(l_{2} \omega+\frac{\omega}{2} k_{2}\right)
$$

Therefore, $\angle H(\omega)-\angle G(\omega)=\left(l_{2}-l_{1}\right) \omega+(\omega / 2)\left(k_{2}-k_{1}\right)=$ $l \omega+(\omega / 2)\left(k_{2}-k_{1}\right)$.

This proposition implies a simple and unique way for choosing the vanishing moments of primal and dual lowpass filters so that the requirement of phase half-sample offset can be satisfied. Supposing the vanishing moments of $h_{0}, \tilde{h}_{0}, g_{0}$, and $\tilde{g}_{0}$ are of $k_{h}, \tilde{k}_{h}, k_{g}$, and $\tilde{k}_{g}$, respectively, it is demanded that

$$
k_{h}-k_{g}=2 l_{1}+1, \quad \tilde{k}_{h}-\tilde{k}_{g}=2 l_{2}+1, \quad l_{1}, l_{2} \in \mathbb{Z} .
$$

In $[1,2]$, Kingsbury had chosen the odd/even-length filter pairs in designing the Hilbert transform pairs wavelet bases. This proposition shows that the choice is unique.

Second, consider the requirements of identical magnitude responses, which have to be approximately achieved by an optimization procedure. Our strategy is to minimize the integral of magnitude response difference between the primary and dual low-pass filters in whole frequency range

$$
\begin{aligned}
\min & \left\{\int_{-\pi}^{\pi}\left\|G_{0}(\omega)-e^{-j \omega / 2} H_{0}(\omega)\right\|^{2} d \omega\right. \\
& \left.+\int_{-\pi}^{\pi}\left\|\widetilde{G}_{0}(\omega)-\tilde{H}_{0}(\omega) e^{j \omega / 2}\right\|^{2} d \omega\right\} .
\end{aligned}
$$

As a result, the design becomes a constrained optimization question as follows: 


$$
\begin{aligned}
& \min \int_{0}^{\pi}\left(\left(2 \cos \frac{\omega}{2}\right)^{2 k_{g}} P_{g}^{2}(\cos \omega)+\left(2 \cos \frac{\omega}{2}\right)^{2 k_{h}} P_{h}^{2}(\cos \omega)\right. \\
& +\left(2 \cos \frac{\omega}{2}\right)^{2 \tilde{k}_{g}} \widetilde{P}_{g}^{2}(\cos \omega)+\left(2 \cos \frac{\omega}{2}\right)^{2 \widetilde{k}_{h}} \widetilde{P}_{h}^{2}(\cos \omega) \\
& -2\left(2 \cos \frac{\omega}{2}\right)^{k_{g}+k_{h}} \cos \left(l_{1} \omega\right) P_{g}(\cos \omega) P_{h}(\cos \omega) \\
& \left.-2\left(2 \cos \frac{\omega}{2}\right)^{\tilde{k}_{g}+\tilde{k}_{h}} \cos \left(l_{2} \omega\right) \widetilde{P}_{g}(\cos \omega) \tilde{P}_{h}(\cos \omega)\right) d \omega, \\
& p_{h}(z) \overline{\tilde{p}_{h}(z)}=P_{K_{h}}\left(\frac{1}{2}-\frac{z+z^{-1}}{4}\right) \\
& +\left(\frac{1}{2}-\frac{z+z^{-1}}{4}\right)^{K_{h}} R_{h}\left(z+z^{-1}\right) \\
& p_{g}(z) \overline{\tilde{p}_{g}(z)}=P_{K_{g}}\left(\frac{1}{2}-\frac{z+z^{-1}}{4}\right) \\
& +\left(\frac{1}{2}-\frac{z+z^{-1}}{4}\right)^{K_{g}} R_{g}\left(z+z^{-1}\right) .
\end{aligned}
$$

In this way, the objective function and constraints are all expressed by the auxiliary filters. The constraints in (16) are equivalent to these in (9) essentially. If the low-pass filters have high vanishing moments in total, the variable number of objective function and constraints in (17) is less than that in (9). For example, the variables in (17) corresponding to the primal filters consist of the coefficients of $p_{h}(z) \tilde{p}_{h}(z)$ and $R_{h}(\cdot)$, whose numbers are $\left(l_{h}+\tilde{l}_{h}+2\right)$ and $\left[l_{h}+\tilde{l}_{h}-\right.$ $\left.\left(k_{h}+\widetilde{k}_{h}\right) / 2+1\right] / 2$, respectively. The total number in (9) is $\left(l_{h}+\tilde{l}_{h}+2\right)+\left\lfloor k_{h} / 2\right\rfloor+\left\lfloor\tilde{k}_{h} / 2\right\rfloor \geq\left(l_{h}+\tilde{l}_{h}+2\right)+\left(k_{h}+\tilde{k}_{h}-2\right) / 2$. Therefor, if $\left(k_{h}+\widetilde{k}_{h}\right)>(2 / 3)\left(l_{h}+\widetilde{l}_{h}+3\right)$, the variable number in (17) is less than that in(9). In Example 1, $h_{0}$ and $\tilde{h}_{0}$ are all of 12 taps with $k_{h}=\tilde{k}_{h}=5$, and $g_{0}$ and $\tilde{g}_{0}$ are, respectively, 13 tap and of 11 taps with $k_{g}=\tilde{k}_{g}=4$. The variable number for the design scheme (17) is 20 however, it is 25 for the design scheme (9). Therefore, the calculation burden in optimization process can be reduced efficiently because of less variables. In fact, the constraints in (9) can be expressed by the auxiliary filters only (without the coefficients of $R_{p}(\cdot)$ and $\left.R_{h}(\cdot)\right)$ therefore, the variable number can be further reduced, and only the expressions become quite complicated.

\section{Design Examples}

Two biorthogonal filter banks are designed, whose corresponding wavelet bases $\psi_{h}(t), \widetilde{\psi}_{h}(t), \psi_{g}(t)$, and $\tilde{\psi}_{g}(t)$ form Hilbert transform pairs, that is, they satisfy (6).

Example 1. In this example, the design process can be described by the following steps.

(1) Selection Vanishing Moments. suppose the vanishing moments of the primal and dual filter banks are $k_{g}, \tilde{k}_{g}, k_{h}$, and $\tilde{k}_{h}$, respectively. $k_{g}$ and $\tilde{k}_{g}\left(k_{h}\right.$ and $\left.\tilde{k}_{h}\right)$ are either both odd or even; it means that $k_{g}+\tilde{k}_{g}=2 N_{1}, k_{h}+\tilde{k}_{h}=2 N_{2}$, and $N_{1}, N_{2} \in \mathbb{Z}^{+}$. According to the proposition in the previous section, it is required that $k_{g}-k_{h}=2 l_{1}+1, \tilde{k}_{g}-\tilde{k}_{h}=2 l_{2}+1$, $l_{1}, l_{2} \in \mathbb{Z}$. In order to make the two filters have equivalent vanishing moments, we select $k_{g}=\widetilde{k}_{g}=4, k_{h}=\widetilde{k}_{h}=5$. We select $L_{h}=\tilde{L}_{h}=12, L_{g}=13, \widetilde{L}_{g}=11$ to make the two filter banks have the equivalent lengths and $R_{g}(\cdot) \neq 0, R_{h}(\cdot) \neq 0$.

(2) Forming Constraints. let the auxiliary filters $p_{h}=\left[p_{h, 3}\right.$, $\left.p_{h, 2}, p_{h, 1}, p_{h, 0}, p_{h, 1}, p_{h, 2}, p_{h, 3}\right], \tilde{p}_{h}=\left[\tilde{p}_{h, 3}, \tilde{p}_{h, 2}, \tilde{p}_{h, 1}, \tilde{p}_{h, 0}, \widetilde{p}_{h, 1}\right.$, $\left.\widetilde{p}_{h, 2}, \widetilde{p}_{h, 3}\right]$. From $(17), R_{h}(\cdot)$ is an odd polynomial of firstdegree. Suppose that $R_{h}(x)=a x$, from (17), we have

$$
\begin{gathered}
p_{h, 3} \tilde{p}_{h, 3}=-1 / 2048 a \\
p_{h, 3} \tilde{p}_{h, 2}+p_{h, 2} \tilde{p}_{h, 3}=5 / 1024 a \\
p_{h, 3} \tilde{p}_{h, 1}+p_{h, 2} \tilde{p}_{h, 2}+p_{h, 1} \tilde{p}_{h, 3}=35 / 128-23 / 1024 a \\
p_{h, 3} \tilde{p}_{h, 0}+p_{h, 2} \tilde{p}_{h, 1}+p_{h, 0} \tilde{p}_{h, 3}+p_{h, 1} \tilde{p}_{h, 2} \\
=65 / 1024 a-175 / 64 \\
p_{h, 3} \tilde{p}_{h, 1}+p_{h, 2} \tilde{p}_{h, 0}+p_{h, 1} \tilde{p}_{h, 1}+p_{h, 0} \tilde{p}_{h, 2}+p_{h, 1} \tilde{p}_{h, 3} \\
=95 / 8-255 / 2048 a, \\
p_{h, 3} \tilde{p}_{h, 2}+p_{h, 2} \tilde{p}_{h, 1}+p_{h, 1} \tilde{p}_{h, 0}+p_{h, 0} \tilde{p}_{h, 1}+p_{h, 1} \tilde{p}_{h, 2}+p_{h, 2} \tilde{p}_{h, 3} \\
=-1825 / 64+93 / 512 a, \\
2 p_{h, 3} \tilde{p}_{h, 3}+2 p_{h, 2} \tilde{p}_{h, 2}+2 p_{h, 1} \tilde{p}_{h, 1}+p_{h, 0} \tilde{p}_{h, 0} \\
=2509 / 64-105 / 512 a .
\end{gathered}
$$

Similarly, the constraints on $p_{g}(z)$ and $\tilde{p}_{g}(z)$ can be formed.

(3) Forming Objective function. the objective function becomes

$$
\begin{gathered}
\int_{-\pi}^{\pi}\left\|G_{0}(\omega)-e^{-j \omega / 2} H_{0}(\omega)\right\|^{2} d \omega \\
+\int_{-\pi}^{\pi}\left\|\widetilde{G}_{0}(\omega)-e^{j \omega / 2} \tilde{H}_{0}(\omega)\right\|^{2} d \omega \\
=2 \int_{0}^{\pi} \cos ^{8}(\omega / 2)\left[\left(P_{g}(\cos \omega)-\cos (\omega / 2) P_{h}(\cos \omega)\right)^{2}\right. \\
\left.+\left(\widetilde{P}_{g}(\cos \omega)-\cos (\omega / 2) \widetilde{P}_{h}(\cos \omega)\right)^{2}\right] d \omega .
\end{gathered}
$$

The objective function (19) is then simplified using the integral function int in Symbolic Math Toolbox in Matlab. The coefficients of auxiliary filters are obtained using the fmincon routine in Matlab. Finally, the filters $h_{0}, g_{0}$, $\tilde{h}_{0}$, and $\tilde{g}_{0}$ are obtained, which are listed in Table 1 . The plots of magnitude spectra $\left|\Psi_{h}(\omega)+i \Psi_{g}(\omega)\right|$ and $2\left|\Psi_{h}(\omega)\right|$, $\left|\tilde{\Psi}_{h}(\omega)+i \tilde{\Psi}_{g}(\omega)\right|$ and $2\left|\tilde{\Psi}_{h}(\omega)\right|$ are depicted in Figure 3 . The magnitude spectra $\left|\Psi_{h}(\omega)+i \Psi_{g}(\omega)\right|$ and $\left|\widetilde{\Psi}_{h}(\omega)+i \widetilde{\Psi}_{g}(\omega)\right|$ each are almost one sided, which show that the wavelets have very good approximation quality to Hilbert transform pairs.

In [13], Tay et al. presented two measures, $E_{1}$ and $E_{2}$, to evaluate the approximation quality to Hilbert transform pair 


$$
\begin{aligned}
& E_{1}=\frac{\max _{\omega<0}\left|\Psi_{h}(\omega)+i \Psi_{g}(\omega)\right|}{\max _{\omega>0}\left|\Psi_{h}(\omega)+i \Psi_{g}(\omega)\right|}, \\
& E_{2}=\frac{\int_{-\infty}^{0}\left|\Psi_{h}(\omega)+i \Psi_{g}(\omega)\right|^{2} d \omega}{\int_{0}^{\infty}\left|\Psi_{h}(\omega)+i \Psi_{g}(\omega)\right|^{2} d \omega} .
\end{aligned}
$$

We employ these measures as criterions in evaluating the design schemes. For the wavelet bases in reconstruction side, $E_{1}$ and $E_{2}$ are defined as the reciprocal of that in decomposition side. The two evaluation measures are given in Table 2.

Example 2. A biorthogonal filter bank (dual filter bank) is designed whose wavelet bases and the wavelet bases of Antonini 9/7 biorthogonal filter bank (primal filter bank) form Hilbert transform pairs. The lengths of dual filter bank are chosen as $10 / 10$ with $k_{g}=\tilde{k}_{g}=3$, which are identical with the choices in [7]. Therefore, the auxiliary filter $p_{g}$ is of 7 taps with four unknown variables, and so is $\widetilde{p}_{g}$. The primal auxiliary filter $p_{h}$ is of 5 taps, whose first three coefficients are $[0.427980118572955,-1.981746369377800$, 4.107532501609684], and $\tilde{p}_{h}$ is of 3 taps with the first two coefficients $[-0.730174104914022,2.460348209828043]$. The filter coefficients we obtained are listed in Table 3. The plots of magnitude spectra $\left|\Psi_{h}(\omega)+i \Psi_{g}(\omega)\right|$ and $2\left|\Psi_{h}(\omega)\right|$ are depicted in Figure $4(\mathrm{a}) ;\left|\widetilde{\Psi}_{h}(\omega)+i \widetilde{\Psi}_{g}(\omega)\right|$ and $2\left|\widetilde{\Psi}_{h}(\omega)\right|$ are depicted in Figure 4(b). $\left|\Psi_{h}(\omega)+i \Psi_{g}(\omega)\right|$ and $\left|\widetilde{\Psi}_{h}(\omega)+i \widetilde{\Psi}_{g}(\omega)\right|$ are all almost one sided, which implies that $\psi_{g}(t) \approx \mathscr{H}\left(\psi_{h}(t)\right)$ and $\tilde{\psi}_{h}(t) \approx \mathscr{H}\left(\tilde{\psi}_{g}(t)\right)$. The measures $E_{1}$ and $E_{2}$ of decomposition and reconstruction wavelets together with their average values are given in Table 2, which shows that the approximation quality of the designed biorthogonal wavelets to Hilbert transform pair has improved to some degree in terms of the average values.

\section{Conclusion}

This paper describes a new scheme for the design of Hilbert transform pairs of biorthogonal wavelets in detail. The relationship between the phase offset and the difference of vanishing moments is useful in choosing the vanishing moments so that the phase requirement on the corresponding low-pass filters can be satisfied strictly. The design is also simplified as a constrained optimization procedure. The objective function and constraints are all expressed in terms of the auxiliary filters, which can reduce the total variable number when the low-pass filters are of high vanishing moments. Consequently, the computational complexity can be reduced efficiently. The objective function expresses the magnitude response requirements in the whole frequency range properly, which is useful in improving the approximation quality to Hilbert transform pairs.

\section{Acknowledgments}

This work has been supported by National Natural Science Foundation of China (NSFC) under Grant no. 60972156 and Beijing Natural Science Foundation under Grant no. 4102017 .

\section{References}

[1] N. G. Kingsbury, "Image processing with complex wavelets," Philosophical Transactions of the Royal Society A, vol. 357, no. 1760, pp. 2543-2560, 1999.

[2] N. G. Kingsbury, "Complex wavelets for shift invariant analysis and filtering of signals," Applied and Computational Harmonic Analysis, vol. 10, no. 3, pp. 234-253, 2001.

[3] F. C. A. Fernandes, I. W. Selesnick, R. L. C. Van Spaendonck, and C. S. Burrus, "Complex wavelet transforms with allpass filters," Signal Processing, vol. 83, no. 8, pp. 1689-1706, 2003.

[4] I. W. Selesnick, "Hilbert transform pairs of wavelet bases," IEEE Signal Processing Letters, vol. 8, no. 6, pp. 170-173, 2001.

[5] H. Ozkaramanli and R. Yu, "On the phase condition and its solution for Hilbert transform pairs of wavelet bases," IEEE Transactions on Signal Processing, vol. 51, no. 12, pp. 32933294, 2003.

[6] R. Yu and H. Ozkaramanli, "Hilbert transform pairs of orthogonal wavelet bases: necessary and sufficient conditions," IEEE Transactions on Signal Processing, vol. 53, no. 12, pp. 4723-4725, 2005.

[7] R. Yu and H. Ozkaramanli, "Hilbert transform pairs of biorthogonal wavelet bases," IEEE Transactions on Signal Processing, vol. 54, no. 6, pp. 2119-2125, 2006.

[8] I. W. Selesnick, "The design of approximate Hilbert transform pairs of wavelet bases," IEEE Transactions on Signal Processing, vol. 50, no. 5, pp. 1144-1152, 2002.

[9] R. Yu, "Characterization and sampled-data design of dual-tree filter banks for Hilbert transform pairs of wavelet bases," IEEE Transactions on Signal Processing, vol. 55, no. 6, pp. 2458-2471, 2007.

[10] I. Daubechies, Ten Lectures on Wavelets, SIAM, Philadelphia, Pa, USA, 1992.

[11] D. Walnut, An Introduction to Wavelet Analysis, Birkhäuser, Boston, Mass, USA, 2002.

[12] A. Cohen, I. Daubechies, and J.-C. Feauveau, "Biorthogonal bases of compactly supported wavelets," Communications on Pure and Applied Mathematics, vol. 45, pp. 485-560, 1992.

[13] D. B. H. Tay, N. G. Kingsbury, and M. Palaniswami, "Orthonormal Hilbert-pair of wavelets with (almost) maximum vanishing moments," IEEE Signal Processing Letters, vol. 13, no. 9, pp. 533-536, 2006. 